\title{
A Theoretical Study of the Production and Decay of Localized Electron Density Enhancements in the Polar Ionosphere
}

\author{
J. J. SOJKA AND R. W. SCHUNK \\ Center for Atmospheric and Space Sciences, Utah State University, Logan
}

\begin{abstract}
The origins, transport, and decay of large-scale $(\gtrsim 10 \mathrm{~km}) F$ region density irregularities were theoretically studied using a high-latitude time dependent ionospheric model. Such density irregularities (blobs) have been found both in the polar cap and the auroral zone. The model study, which focuses on blobs being produced by auroral precipitation, shows that the observed energy fluxes can readily account for the blob densities if a plasma flux tube is exposed to the precipitation for $5-10 \mathrm{~min}$. Once the flux tube is transported away from the source, the $F$ region density profile recovers its shape on a time scale of $10-20 \mathrm{~min}$. Hence after this time period it is not possible to determine whether hard or soft precipitation was the source of the blob. Once created a blob will maintain its relative size for many hours, since it decays at the same rate as the background plasma. Blobs are removed by being transported into a region of high production, where the new background density exceeds the old blob density. The high $h_{m} F_{2}$ 's observed with some blobs need not necessarily imply soft precipitation; induced upward plasma drifts from neural winds or ExB convection can readily account for them. Since the high latitude ionosphere contains discrete auroral forms in the oval and polar cap and since plasma is continually convecting through these regions, blobs are the rule rather than the exception.
\end{abstract}

\section{INTRODUCTION}

Large-scale $F$ region density irregularities on the scale of 10 's to 100 's of km have been frequently observed in the high-latitude ionosphere [Vickrey et al., 1980; Kelley et al., 1982; Kelley and Vickrey, 1984; Weber et al., 1984; Foster and Doupnik, 1984; de la Beaujardiere et al., 1985]. These density irregularities, more commonly referred to as blobs, are predominantly an $F$ region peak and topside phenomenon. They have been observed in the dayside cusp [Kelley and Vickrey, 1984; Foster and Doupnik, 1984], auroral zone [Vickrey et al., 1980; Robinson et al., 1985], and in the polar cap [Weber et al., 1984; de la Beaujardiere et al., 1985]. Several blob source mechanisms have been proposed: very soft precipitation, which leads to high-altitude ionization [Kelley et al., 1982]; convection of plasma over large distances from an auroral source to the observed location [Knudsen, 1974; Weber et al. 1984); and solar EUV-produced ionization convected across the polar cap with the lower densities between blobs caused by enhanced plasma loss rates [de la Beaujardiere et al., 1985]. Kelley and Vickrey [1984] suggest that these enhanced loss rates could result from structure in the convection electric field; regions of high electric field lead to enhanced loss of $\mathrm{O}^{+}$via a chemical conversion of $\mathrm{O}^{+} \rightarrow \mathrm{NO}^{+}$. All three mechanisms could account for the observed blob characteristics; which is the dominant mechanism or what their relative importance is has not been determined. Kelley et al. [1982] studied the sources and decay of irregularities and they describe in general terms how the larger scale irregularities $(\gtrsim 10 \mathrm{~km})$ are produced by soft precipitation and how the smaller scale size blobs of dimensions much less than a kilometer are produced within the larger blobs through plasma instabilities.

In this study we focused attention on these larger "parent" blobs. Our high-latitude time-dependent $F$ region model (HLTD) [Schunk and Raitt, 1980; Sojka et al., 1981a, b; Sojka and Schunk, 1985] was used to simulate the production, convection, and decay of ionospheric plasma irregularities. For this

\section{Copyright 1986 by the American Geophysical Union.}

Paper number 5A8654

0148-0227/86/005A-8654\$05.00 study we considered discrete auroral forms as the source of the blobs. Discrete auroral forms in the cleft, polar cap, and auroral oval are well documented, thereby enabling us to realistically model them. Structures in the convection electric field also exist. However, our understanding of these is much poorer, and hence for this study we used uniform convection patterns. Several precipitating particle spectra, which produce different ionization profiles, were used as source mechanisms for the parent blobs. An important goal of this study was to determine if soft precipitation is a necessary ingredient for blob formation. The effects of both plasma decay and convection were contrasted to determine the time scales for blob persistence. In an earlier study [Sojka and Schunk, 1985] we used our HLTD model to determine the effectiveness of neutral wind induced upward plasma drifts in maintaining the $F$ layer in the night sector. These upward drifts, especially during solar maximum conditions, can lead to night sector $h_{m} F_{2}$ values in excess of $400 \mathrm{~km}$. Such high $h_{m} F_{2}$ 's are also associated with night sector blob observations [cf. de la Beaujardiere et al., 1985]. To date, these high $h_{m} F_{2}$ values have been interpreted as evidence of soft precipitation, which leads to high-altitude ion production. In this study we did not attempt to correlate high $h_{m} F_{2}$ values with soft precipitation; our earlier study indicates that these high $h_{m} F_{2}$ 's can be produced separately by induced upward plasma drifts.

\section{Auroral Plasma Enhancement}

The importance of auroral particle precipitation in producing "local" $F$ region ionization enhancements has been clearly demonstrated [Rees, 1963; 1969; Rees and Maeda, 1973; Banks et al., 1974; Knudsen, 1974]. On a global scale, Knudsen et al. [1977] simulated these effects by allowing convection to transport plasma flux tubes through regions of enhanced auroral ionization. In our global studies of the high latitude ionosphere, we used a statistical auroral energy flux model and simply scaled a single set of ionization rate profiles in order to obtain auroral production rates [Sojka et al., 1981a, b; Sojka and Schunk, 1983].

In this study we considered the effects of a set of discrete auroral structures as well as different precipitation ionization rate profiles. Figure 1 shows the four $\mathrm{O}^{+}$production rate profiles 


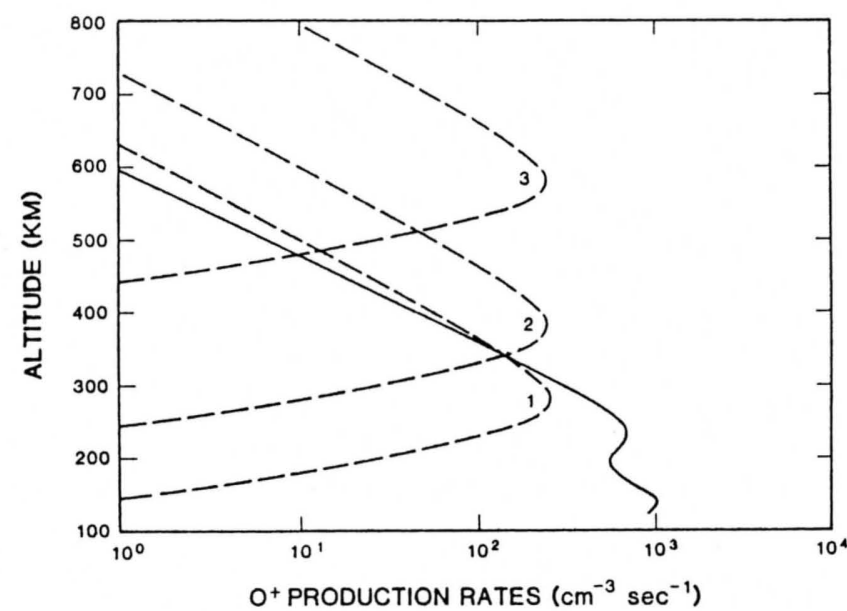

Fig. 1. $\mathrm{O}^{+}$production rates as a function of altitude. The solid curve corresponds to the $\mathrm{O}^{+}$production rate for a typical hard auroral spectrum, while the dashed curve (1) corresponds to a soft cusp spectrum. The dashed curves 2 and 3 are the same as curve 1, except that the profile has been raised to higher altitudes.

used in this study. The solid line is for a hard auroral precipitation spectrum. This profile is identical to that used in our earlier studies and comes from Knudsen et al. [1977]. The profile corresponds to an auroral energy flux of $0.92 \mathrm{ergs} /\left(\mathrm{cm}^{2} \mathrm{~s} \mathrm{sr}\right)$. We also used the corresponding $\mathrm{O}_{2}^{+}$and $\mathrm{N}_{2}^{+}$production rate profiles given by $\mathrm{Knudsen}$ et al. Soft, subkilovolt, auroral $\mathrm{O}^{+}$production is represented by the dashed profile labelled 1 . This profile corresponds to the cusp precipitation profile of Knudsen et al. and has an associated auroral energy flux of $0.20 \mathrm{ergs} /\left(\mathrm{cm}^{2} \mathrm{~s} \mathrm{sr}\right)$. The average primary energy of the soft and hard auroral spectra are 0.2 and $2 \mathrm{KeV}$, respectively.

In addition to the auroral spectrum, the neutral atmosphere is critical in determining the ion production rates. Knudsen et al. [1977] used a neutral atmosphere that is appropriate for medium solar activity. In this study we considered solar maximum conditions, a time when auroral ionization enhancement are frequently observed. With an enhanced solar maximum neutral temperature, the neutral scale height and hence neutral densities are increased. With the higher neutral densities, the soft precipitating electrons are unable to penetrate as deeply into the atmosphere as they do for medium solar activity. As a result, the associated ion production occurs at a higher altitude at solar maximum. To simulate this effect we simply raised the "soft" $\mathrm{O}^{+}$production rate profile 1 by $100 \mathrm{~km}$ and thereby obtained a solar maximum production rate profile (curve 2 in Figure 1); $100 \mathrm{~km}$ is about the maximum difference between solar minimum and maximum $h_{m} F_{2}$ values [Sojka and Schunk, 1985]. The final dashed curve 3 in Figure 1 is intended to represent a hypothetical case of $\mathrm{O}^{+}$production due to extremely high fluxes of very cold precipitating electrons $(\sim 50 \mathrm{eV})$. This curve was obtained by raising curve 2 by $200 \mathrm{~km}$. There is no specific auroral spectrum or energy flux available for either curves 2 or 3 . However, these profiles were used to study the $F$ region's response to high-altitude ionization.

To study the effect of enhanced ionization and convection in the polar cap, a single plasma flux tube was selected. Figure 2 (top right) shows part of the flux tube trajectory, from 1200 to 2400 MLT. After midnight this flux tube essentially corotates back to noon. Each of the dots composing the trajectory represents the location at which we stored ion density profiles calculated by our high latitude ionospheric model. Between steps 16 and 20 , the trajectory passes through the region of enhanced auroral ion production. This region can be schematically viewed as the cleft. In the lower part of Figure 2 we show how the precipitating energy flux varies during the auroral crossing. This figure also relates the trajectory step number to a relative time scale, which is referenced to a point just prior to the flux tube entering the auroral region. The flux tube convects through the strongest cleft precipitation in under 10 minutes. This whole procedure follows from the original suggestion of Knudsen [1974] on how blobs could be formed and convected across the polar cap to the nightside.

The trajectory shown in Figure 2 was obtained from a duskenhanced Volland-type convection model whose cross-polar cap potential was $62 \mathrm{kV}$. In the polar cap the electric field was uniform at $15 \mathrm{mV} / \mathrm{m}$, while equatorward of this region the potential diminished as the inverse of the sine of magnetic colatitude to the fourth power. The polar cap, which was a circle with a radius of $17.9^{\circ}$, was offset in the antisunward direction by $2.2^{\circ}$ from the magnetic pole. This convection pattern corresponds to moderately active conditions with a $K p$ of 3 .

In addition to a convection pattern, our HLTD model requires a neutral atmosphere and wind pattern. The mass spectrometer/incoherent scatter model for solar maximum $(F 10.7=150$ and $A p=15)$ and winter seasonal conditions was used. For the neutral wind we adopted the same pattern used by Sojka et al. [1981a]. This wind blows across the polar cap from 1300 to $0100 \mathrm{LT}$, attaining a maximum $F$ region speed of 200 $\mathrm{m} / \mathrm{s}$. On the dayside the wind speed is small, and for this study it was set to zero. The $\mathrm{O}^{+}$ion energy equation, allowing for the electric field, was solved to obtain ion temperatures. In contrast, the electron temperature was assumed. Three different temperature profiles were used: a dayside, a nightside, and an oval temperature profile [see Sojka et al., 1981a].

The location of the dayside oval (Figure 2) corresponds to dipole latitudes between $75^{\circ}$ and $78^{\circ}$. This location is consistent with observations of the dayside aurora [Meng, 1981]. For the purposes of this part of the study, no evening sector auroral oval was included. In the dayside auroral oval, various types of auroral precipitation spectra are found. They fall into two main categories, soft with energies below $\sim 200 \mathrm{eV}$ and hard with

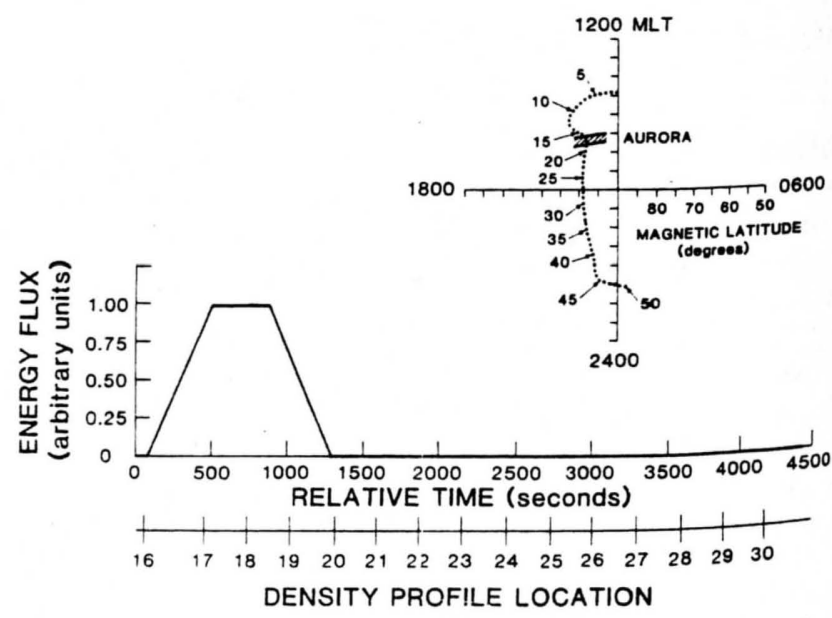

Fig. 2. Test trajectory for plasma transport across the polar cap in a magnetic latitude-MLT reference frame (top). Density profile locations have been numbered along the trajectory, and the cross-hatch region corresponds to an auroral ionization source. The bottom part of the figure shows the relation between elapsed time, density profile location, and auroral energy flux. 
TABLE 1. Selected Auroral Production Models

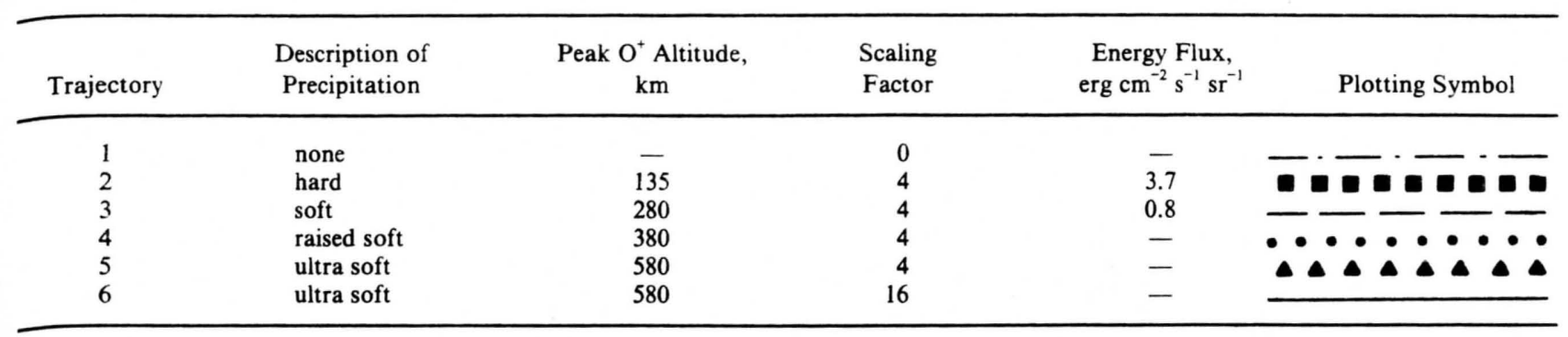

typical energies $\gtrsim 2 \mathrm{KeV}$ (see Meng [1981], and references therein). The energy flux of the soft precipitation is usually below 1 $\mathrm{erg} \mathrm{cm}{ }^{-2} \mathrm{~s}^{-1} \mathrm{sr}^{-1}$, but in restricted auroral regions can reach a few ergs $\mathrm{cm}^{-2} \mathrm{~s}^{-1} \mathrm{sr}^{-1}$. For the hard precipitation, the energy fluxes

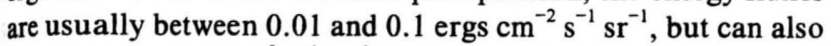
reach a few ergs $\mathrm{cm}^{-2} \mathrm{~s}^{-1} \mathrm{sr}^{-1}$ [Meng, 1981].

In Table 1 the various auroral precipitation spectra used for six otherwise identical trajectory runs are shown. For the first trajectory run, no auroral precipitation was included, and consequently, this run can be used as a baseline against which comparisons can be made. Trajectory runs 2 and 3 correspond to the hard and soft (1) production profiles shown in Figure 1, which have been scaled up to give energy fluxes of 3.7 and 0.8 $\mathrm{ergs} \mathrm{cm}^{-2} \mathrm{~s}^{-1} \mathrm{sr}^{-1}$, respectively. Similarly, trajectory runs 4 and 5 correspond to the soft 2 and 3 production profiles shown in Figure 1, with the energy fluxes scaled by a factor of 4 . The final trajectory run 6 is identical to trajectory run 5 , except that the energy flux has been scaled by a further factor of four. Table 1 gives the line symbols which are used to distinguish between the six trajectory runs in Figures 3, 4, and 5. Also included in Table 1 are the altitudes of the peak $\mathrm{O}^{+}$production. Both the hard and soft ( 2 and 3) profiles have their peaks below the nominal $F$ region peak $(\sim 350 \mathrm{~km})$. The results of following the trajectory shown in Figure 2 are contrasted for each of the dayside auroral ionization sources of Table 1 in the following paragraphs.

Figure 3 shows eight sets of $N_{e}$ profiles for the six different auroral ionization sources given in Table 1 . In each panel the step number and relative time of the profiles along the Figure 2 trajectory are given. The top left panel shows the $N_{e}$ profiles at step 16, just prior to entering the auroral region. All six profiles are identical at this time. The profile obtained just prior to entering the oval dependents on several factors, including UT, dayside wind-induced vertical drift, convection pattern, season, solar cycle, etc. The Figure 2 trajectory carries solar EUV produced ionization across the polar cap, a feature referred to as a tongue of "ionization" [see Knudsen, 1974]. More recently, the marked UT dependence of this transpolar plasma convection has been observed by de la Beaujardiere [1985]. These UT and seasonal dependencies in the solar produced ionization are treated in this study as a background upon which precipitationinduced blobs are created. The Figure 2 trajectory was started on the noon magnetic meridian at $0443 \mathrm{UT}$, and it takes 13 hours, $56 \mathrm{~min}$ to traverse the complete trajectory. A total of 3 hours, 38 min elapsed in reaching step 16 . The $N_{e}$ profile at this step has an $h_{m} F_{2}$ of $370 \mathrm{~km}$ and an $N_{m} F_{2}$ of $2 \times 10^{5} \mathrm{~cm}^{-3}$ and shows a marked absence of $E$ region ionization, a result of having crossed the solar terminator into darkness.

Step 18 (top right panel, Figure 3) shows $N_{e}$ profiles inside the auroral region. Since this is only $11 \mathrm{~min}$ after step 16 , it is apparent that $N_{e}$ quickly responds to increased auroral ioniza- tion. These profiles are not steady state profiles. The hard precipitation (squared line) produces a pronounced $E$ region whose density of about $3 \times 10^{5} \mathrm{~cm}^{-3}$ exceeds the other $E$ region densities by over an order of magnitude. All the $N_{e}$ profiles are enhanced over the profile which corresponds to the run with no precipitation (dashed-dotted line), and their respective peaks lie close to the altitude of peak production (see Table 1). Profiles 5 and 6 (triangular line and solid curve) have peaks well above the normal $F$ peak: a consequence of high-altitude production in combination with downward diffusion.

Step 20 occurs about 9 min after step 18 and lies just beyond the auroral region (Figure 2). Already there is a marked decrease in density at low altitudes for the hard precipitation case (about a factor of four at $150 \mathrm{~km}$; our model is rigorously valid only above this altitude in the dark hemisphere). Near $150 \mathrm{~km}$ the ionosphere is predominantly maintained by downward diffu-

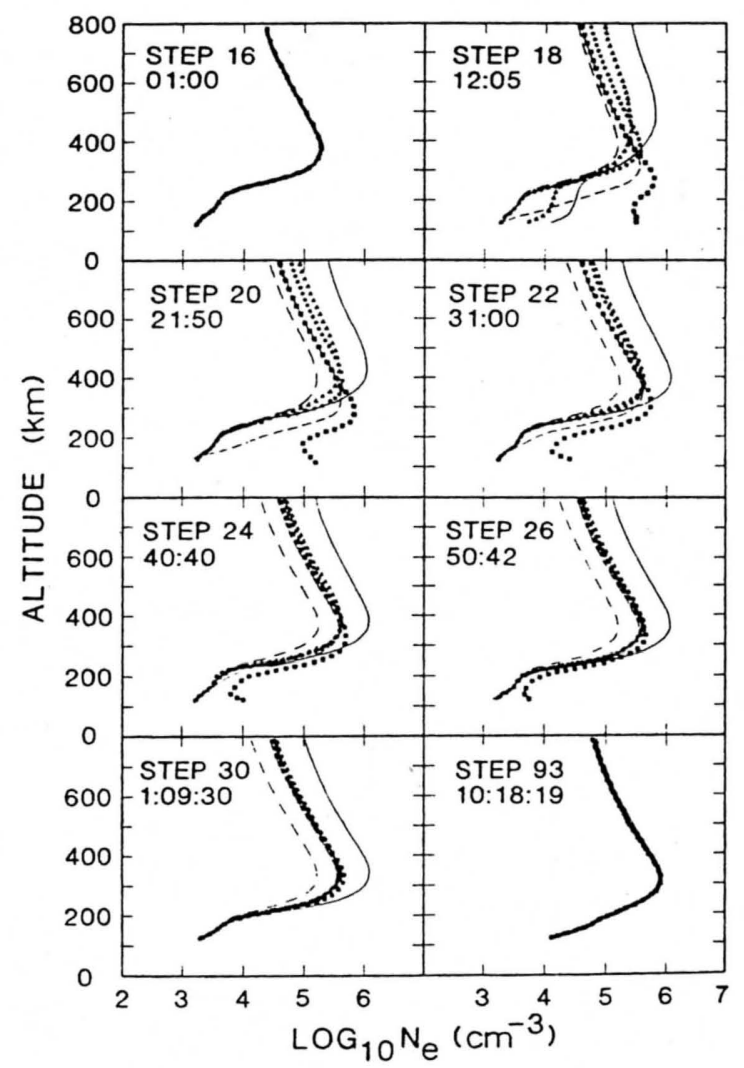

Fig. 3. Electron density profiles at selected locations along the test trajectory for the different auroral production models defined in Table 1. The elapsed times are given in (minutes: seconds) below the location number. 
sion in the region outside direct auroral precipitation. Also, the $h_{m} F_{2}$ values for the two high altitude production cases have dropped from $\sim 480 \mathrm{~km}$ at step 18 to about $420 \mathrm{~km}$ in the 100-200 s since the flux tube left the auroral region.

After a further $10 \mathrm{~min}$ (step 22), downward diffusion has brought all the soft precipitation profile $h_{m} F_{2}$ 's to within about $10 \mathrm{~km}$ of the nominal, no precipitation $h_{m} F_{2}$ value. Also, $h_{m} F_{2}$ for the hard precipitation case has increased from $\sim 200$ to 310 $\mathrm{km}$ due to the enhanced bottomside loss rates for these elevated ion densities. All the profiles now have a similar shape on the topside. After $10 \mathrm{~min}$, the topsides have attained approximately the same scale height, indicating how effective diffusion is at removing high altitude density enhancements. Indeed, for the soft precipitation case, where the peak production rate lies above the $F$ region peak, an elevated $h_{m} F_{2}$ is sustained only when the flux tube lies within the soft precipitation region or has only just left the region (a few minutes at most).

Twenty minutes after leaving the precipitation region (step 24) all the profiles, except for the hard precipitation one, have indistinguishable shapes. By step 26 , and most certainly by step 30 , all the profiles would be indistinguishable as far as their original precipitation source characteristics are concerned. Thus, within significantly less than an hour, the $F$ region density profiles show no trace of the precipitation characteristics. However, the values of $N_{m} F_{2}$ are different, since they depend on whether the peak production rate was below or above the nominal $h_{m} F_{2}$ value and on the total energy flux.

After step 30, which lies inside the polar cap, the flux tube drifts in an antisunward direction toward the nocturnal auroral oval, which it crosses between steps 40 and 60 , and then drifts in a corotating direction back to noon with an enhanced velocity. The relative difference between the profiles shown at step 30 is maintained in the night sector even as the overall density decays. However, when the plasma flux tube reaches the dayside terminator and fresh ion production occurs, these old density differences are too small in absolute terms to be discernable from the new dayside plasma densities. By step 93 , which is at magnetic noon and 9 hours and 9 min after step 30 , solar EUV production completely dominates the $N_{e}$ profiles, and all six profiles are identical.

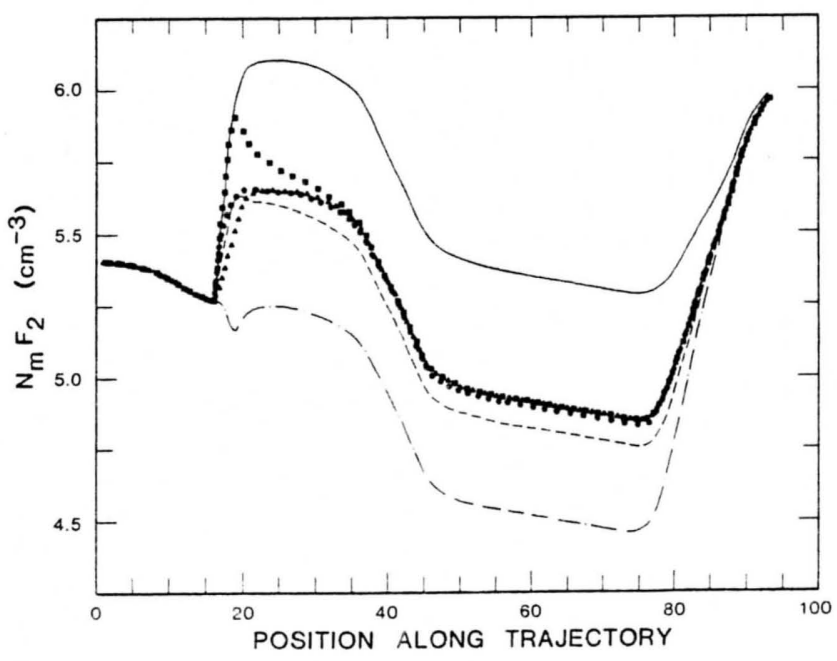

Fig. 4. $N_{m} F_{2}$ variation along the test trajectory for the different auroral production models defined in Table 1 .

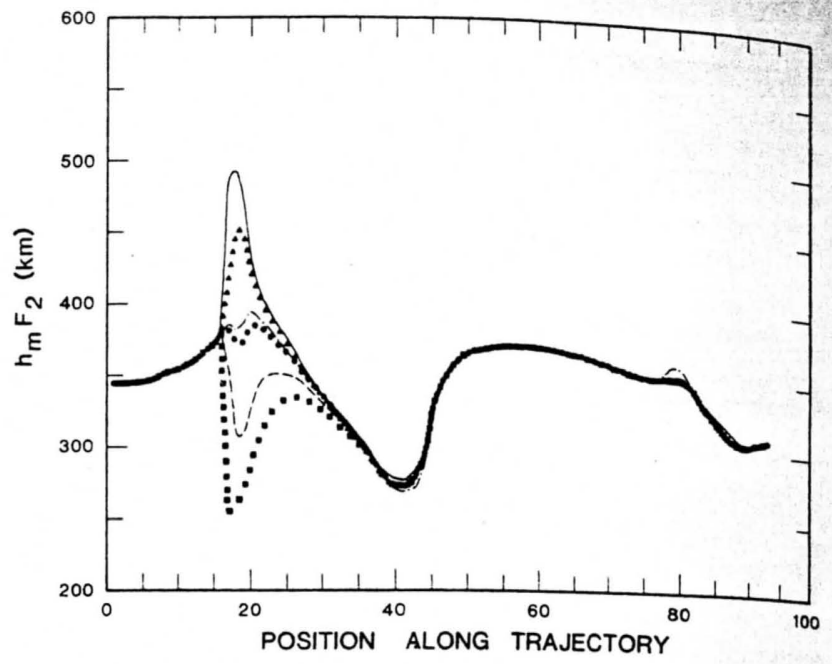

Fig. 5. $h_{m} F_{2}$ variation along the test trajectory for the different auroral production models defined in Table 1 .

Figure 4 shows how $N_{m} F_{2}$ varies around the trajectory for the six cases. Prior to entering the dayside oval (step 16), the $N_{m} F_{2}$ variations for the six cases are the same. In the oval (between steps 16 and 20), the $N_{m} F_{2}$ variations are different because the six flux tubes are subjected to different ionization sources. Also, for a short period of time after the flux tubes leave the precipitation region, the $N_{m} F_{2}$ variations are different (between steps 20 and 30); this is the time it takes for the flux tubes to adjust to the absence of precipitation. However, when the flux tubes are in darkness (steps 30-80), the $N_{m} F_{2}$ variations are the same. Only after the flux tubes enter sunlight (step 80) do the $N_{m} F_{2}$ values merge together. This indicates that any dayside blob that reaches the terminator before being destroyed will maintain its size relative to the background plasma throughout the night. Likewise, any blob created on the nightside will be maintained. Only when the blob and the background plasma enter a region of strong uniform production will the blob disappear. Of course, a nonuniform or rapidly varying convection pattern will modify our arguments to some extent.

Figure 5 shows how $h_{m} F_{2}$ varies around the trajectory for the six cases. The important feature to note is that only in a very restricted region are the $h_{m} F_{2}$ values different (between steps 16 and 30). In the oval (steps 16-20), the $h_{m} F_{2}$ variations are different because the six flux tubes are subjected to ionization sources that peak at different altitudes. The hard precipitation source produces a low $h_{m} F_{2}(\sim 250 \mathrm{~km})$, while the soft precipitation source that peaks at high altitudes produces a high $h_{m} F_{2}(\sim 500$ $\mathrm{km})$. However, once the flux tubes leave the precipitation region, $h_{m} F_{2}$ quickly readjusts to its nominal value determined by diffusion and induced vertical drifts. Indeed, by about step 25 , which is only $25 \mathrm{~min}$ after the flux tubes leave the auroral region, the $h_{m} F_{2}$ variations are nearly the same.

Note that beyond step 30, the $h_{m} F_{2}$ values are the same for all six cases even though the $N_{m} F_{2}$ values are different. This indicates that outside the source region, $N_{m} F_{2}$ and $h_{m} F_{2}$ are not necessarily related. Once a blob is created, temporal and spatial changes in the convection pattern and/or thermospheric wind will affect $h_{m} F_{2}$ even if $N_{m} F_{2}$ doesn't change. Consequently, the fact that a blob is measured with a high $h_{m} F_{2}$ is not necessarily indicative of its source if the blob is outside of its source region [de la Beaujardiere et al., 1985]. 


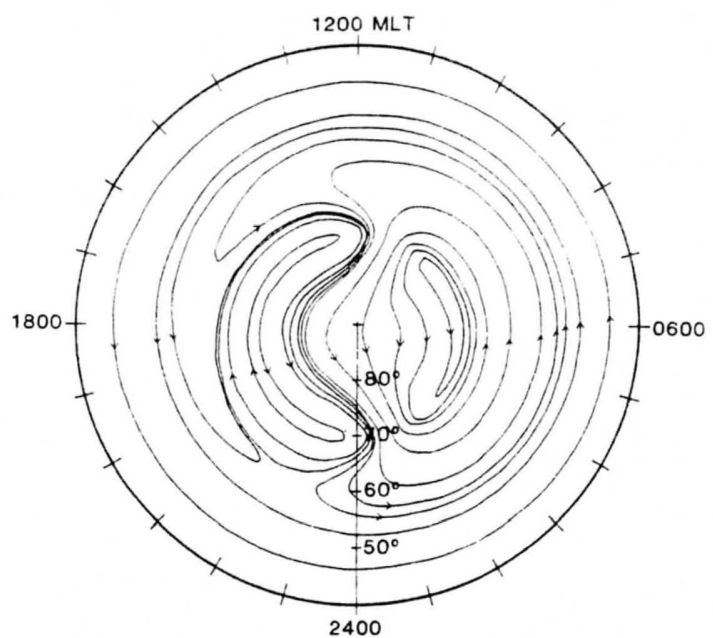

Fig. 6. Plasma flow trajectories in a magnetic latitude-MLT reference frame for a Heelis et al. [1982] convection pattern with corotation added. The convection pattern is a symmetric two-cell pattern with a cross polar cap potential of $75 \mathrm{kV}$.

\section{Blob Simulation}

In this section we describe a simulation that produces ionization patches (blobs) that are consistent with observations. For this simulation we assumed that the blobs were created on the dayside due to the ionization associated with sun-aligned arcs. Such sun-aligned arcs have been observed well into the polar cap [Weber and Buchau, 1981]. The subsequent transport of plasma flux tubes into, across, and out of the polar cap was then followed in order to determine the evolution of the blobs. The sun-aligned arc features and the convection pattern were chosen to be consistent with observations. The density variations obtained from the simulation are shown to be qualitatively consistent with those measured by the Chatanika incoherent scatter radar.

Figure 6 shows the adopted plasma convection pattern in an MLT-dipole latitude frame. The convection pattern was obtained from the Heelis et al. [1982] model. A polar cap potential drop of $75 \mathrm{kV}$ was used, with 45 and $30 \mathrm{kV}$ across the dusk and dawn sectors, respectively. For this model, the highest electric fields are in the afternoon sector of the dayside throat, which has a $45 \mathrm{kV}$ potential drop over 1 hour of MLT. A similar electric field is present post midnight at $70^{\circ}$ magnetic latitude. The convection pattern has a polar cap radius of $18^{\circ}$, is shifted antisunward from the magnetic pole by 2 degrees, and has an equatorward potential which diminishes as the inverse of the sine of magnetic colatitude to the fourth power.

This convection model, together with the appropriate Spiro et al. [1982] auroral precipitation model for a Kp of 4, were used to calculate ionospheric densities for the solar maximum, winter conditions described in section 2. For our initial simulation, discrete auroral forms were not included and only the smooth, statistical auroral oval of Spiro et al. [1982] was considered. Figure 7 (dashed curves) shows the contours of precipitating energy flux for this statistical oval in an MLT-magnetic latitude frame. The ionospheric densities obtained from this initial simulation will be used as a baseline against which the blob simulations can be compared.

Discrete arcs are introduced by having precipitation associated with individual plasma flux tube trajectories. Five such polar cap trajectories are shown in Figure 7. Each trajectory has a thick line segment labelled from one to five. These thick line segments represent regions where polar cap precipitation is introduced to simulate "sun-aligned" arcs. This discrete arc structure follows the suggestion of Reiff et al. [1978] who conclude that the "fan-shaped" distribution of dayside polar cap arcs lies along the throat convection equipotentials described by Heelis et al. [1976]. The arcs shown in Figure 7 are morphologically consistent with the observed satellite images [Snyder and Akasofu, 1976; Meng and Akasofu, 1976] and all sky camera observations of Weber and Buchau [1981].

Table 2 gives the auroral ion production features associated with each of the five discrete arcs shown in Figure 7. Arcs 1 and 4 are of the hard type shown in Figure 1 (solid line) but scaled by factors of 7 and 3, respectively; arc 2 is of the soft type (Figure 1, dashed curve (1)) but scaled by a factor of 5; arc 3 is of the soft type but raised $100 \mathrm{~km}$ (Figure 1, dashed curve (2)) and scaled by a factor of 4; and arc 5 is of the soft type but raised $300 \mathrm{~km}$ (Figure 1, dashed curve (3)) and scaled by a factor of 5 . These scaling factors, although arbitrary, yield corresponding energy fluxes of 6.4 and $2.7 \mathrm{erg} \mathrm{cm}^{-2} \mathrm{~s}^{-1} \mathrm{sr}^{-1}$ for the hard precipitation cases and $1.0 \mathrm{erg} \mathrm{cm}^{-2} \mathrm{~s}^{-1} \mathrm{sr}^{-1}$ for the soft precipitation cases. This is consistent with the observations described earlier [Meng, 1981; Foster and Burrows, 1976]. For this study the five sunaligned arc lengths, energy fluxes, and characteristic energies have been randomly selected.

The five trajectories illustrated in Figure 7 were followed with our HLTD model for all UT's to complement the initial simulation run described above. By comparing the densities with and without the discrete arcs, the effect of the discrete arcs on the densities far from the source region can be studied. Consequently, inferences concerning the role of transport on "blob" observations can also be made.

Figure $8 a$ shows model contours of $\log _{10} N_{e}$ as a function of altitude and magnetic local time (MLT) for a corotating observer on the Chatanika geographic meridian $\left(212^{\circ} \mathrm{E}\right)$ at a dipole latitude of $70^{\circ}$. This location was chosen because the Chatanika incoherent scatter radar had observed "blobs" of plasma convecting equatorward from the polar cap around magnetic midnight [Vickrey et al., 1980; de la Beaujardiere et al.,

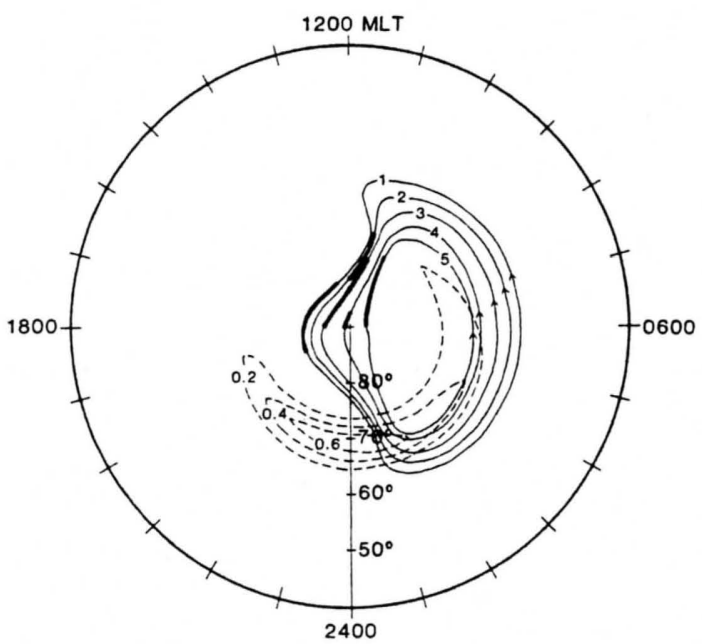

Fig. 7. Five transpolar flow trajectories selected from the adopted convection model. The thick dark curves represent regions of enhanced polar cap auroral precipitation, as defined in Table 2. The dashed curves represent the Spiro et al. [1982] empirical auroral energy flux model. The energy flux is in units of erg cm $\mathrm{cm}^{-2}$. 
TABLE 2. Discrete Arc Spectral Types

\begin{tabular}{lllll}
\hline Arc & $\begin{array}{c}\text { Description of } \\
\text { Precipitation }\end{array}$ & $\begin{array}{c}\text { Figure 1 } \\
\text { Type }\end{array}$ & $\begin{array}{c}\text { Figure 1 } \\
\text { Scaling Factor }\end{array}$ & $\begin{array}{c}\text { Energy Flux, } \\
\mathrm{erg} \mathrm{cm}^{-2} \mathrm{~s}^{-1} \mathrm{sr}^{-1}\end{array}$ \\
\hline 1 & hard & solid line & 7 & 6.4 \\
2 & soft & dashed line (1) & 5 & 1.0 \\
3 & dashed line (2) & 4 & - \\
4 & raised soft & solid line \\
5 & hard & dashed line (3) & 5 & - \\
\hline
\end{tabular}

1985] and convecting poleward into the polar cap in the noon sector [Foster and Doupnik, 1984]. The densities shown in Figure $8 a$ are for the HLTD simulation with no discrete arcs. For different longitudes the density variation will be different due to the inherent UT dependence of the $F$ region which is incorporated in our model. The most pronounced feature in Figure $8 a$ is the $F$ region minimum in $N_{e}$ immediately after midnight. These low densities occur in a high electric field region (see Figure 6 and our earlier description of the convection model). This high electric field gives rise to strong downward induced plasma drifts. Hence around midnight the corotating radar crosses plasma flux tubes moving equatorward which contain densities that are depleted by the strong downward drifts which lower the $F$ layer into a region of higher recombination rates.

From Figure 7 it is evident that the five trajectories which contain discrete arcs all lie within a 1-hour local time sector at $70^{\circ}$ magnetic latitude. Figure $8 b$ shows the model contours of $\log _{10} N_{e}$ as a function of altitude and MLT on the Chatanika geographic meridian at $70^{\circ}$ magnetic latitude for the HLTD simulation that includes the dayside sun-aligned arcs. The trajectories containing the arcs are identified by the labels $X_{1}$ through $X_{5}$. Each label corresponds exactly to the label numbers used in Table 2 as well as in Figure 7. Bearing in mind that each blob is represented by a single trajectory which crosses the corotating radar's MLT track at one point in the night sector, the widths associated with these blobs are purely schematic. However, the density enhancements associated with the blobs is quantitatively correct. Blobs $X_{1}, X_{3}$, and $X_{5}$ are enhanced by about a factor of 2 over the background density. These are the blobs associated with the longest arcs (see Figure 7) and hence had the greatest plasma production. Note, however, that the three arcs had quite different spectral characteristics (see Table 2). The similarity in these three blob $N_{m} F_{2}$ values is purely fortuitous. All five blobs have profile shapes that are the same and they are the same as the nonblob density profile shapes. The $h_{m} F_{2}$ values for all five blobs are also the same (see plus symbols at the center of each blob). This value of $h_{m} F_{2}$ is also the same as the background $h_{m} F_{2}$ on either side of the blobs. Clearly, the ionization source nature of these blobs cannot be inferred from Figure $8 b$ as the effect of transport out of the polar cap has completely removed any source signatures. Based upon reasonable energy fluxes and arc lengths, the blobs generated in Figure $8 b$ show a density enhancement which varies from a few percent to over a factor of 2 relative to the background densities.

Figure $9 a$ is similar to Figure $8 a$, except that the density contours are for a corotating observer at $80^{\circ}$ magnetic latitude on the Chatanika geographic meridian. Note that the diurnal density variation is somewhat different from that at $70^{\circ}$ magnetic latitude (Figure 8a). Since the convection pattern is shifted antisunward by $2^{\circ}$, the "high electric field" flux tube, which is associated with low densities in Figure $8 a$, is now encountered in the 1400 to 1900 MLT region. The flux tubes which move antisunward across the $80^{\circ}$ latitude toward $70^{\circ}$ have their $N_{m} F_{2}$ values decreased by a factor of 2 in this model study. This decrease is not only a result of normal plasma decay, but also a result of enhanced recombination due to the strong downward. induced drifts in this region.

Figure $9 b$ shows the model density contours at $80^{\circ}$ magnetic latitude on the Chatanika geographic meridian for the HLTD

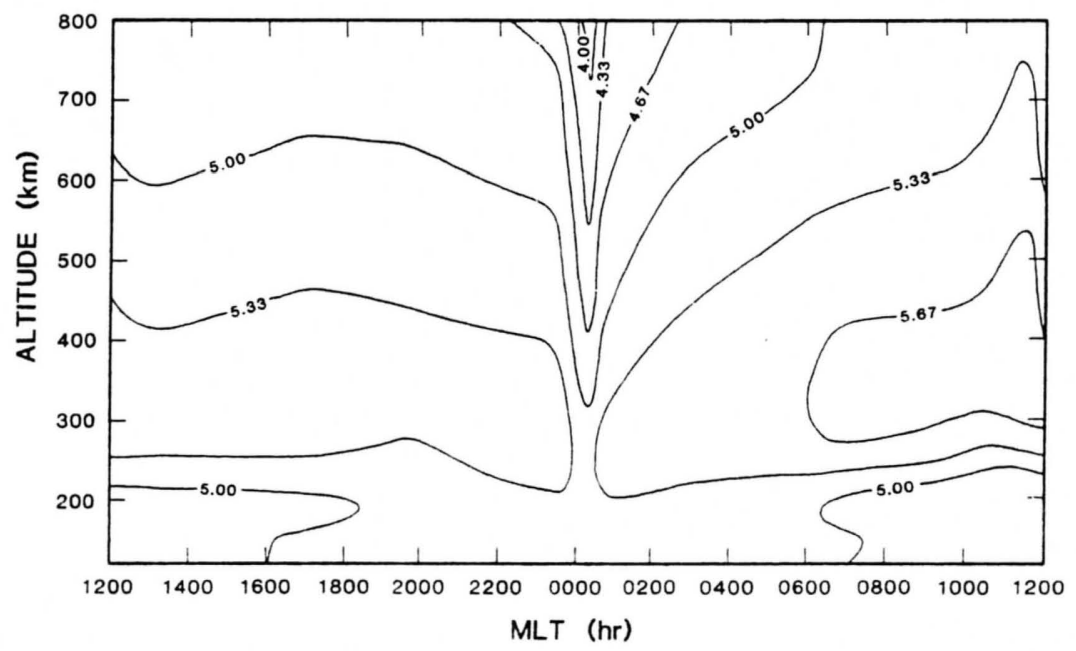

Fig. 8a. Contours of $\log _{10} N_{e}\left(\mathrm{~cm}^{-3}\right)$ as a function of altitude and MLT for the Chatanika geographic meridian $\left(212^{\circ} \mathrm{E}\right)$ but at a dipole latitude of $70^{\circ}$. This is the pattern that occurs in the absence of enhanced polar cap auroral precipitation. 


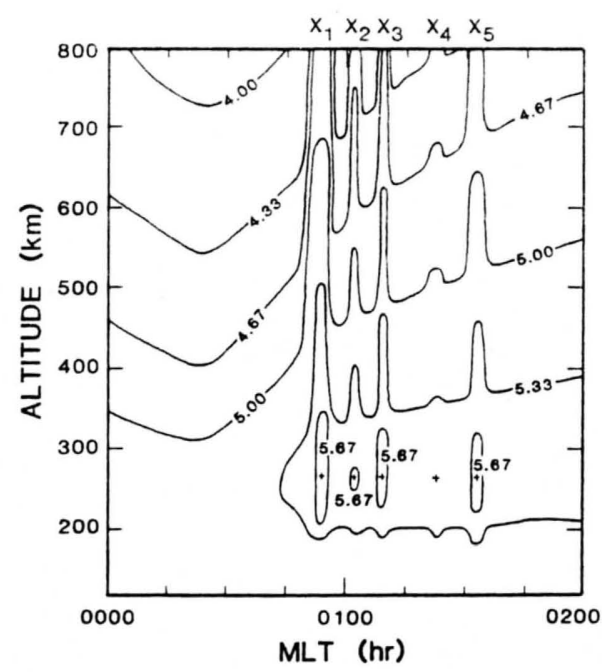

Fig. $8 b$. Contours of $\log _{10} N_{e}\left(\mathrm{~cm}^{-3}\right)$ as a function of altitude and MLT (between 0000 and 0200 hours) for the same location as in Figure $8 a$. For these calculations the enhanced polar cap auroral precipitation shown in Figure 7 has been included. The labels $X_{1}$ to $X_{5}$ identify five density enhancements (blobs or patches). The crosses indicate the values of $h_{m} F_{2}$ in the density enhancements.

simulation that includes the dayside sun-aligned arcs. The blobs are labelled identically to Figure $8 b$. From Figure 7 it is evident that at $80^{\circ}$ the spacing between the blobs is greater than at $70^{\circ}$, and therefore the blob widths in Figure $9 b$ should be somewhat larger than in Figure $8 b$. However, as noted earlier, only a single flux tube is associated with each blob, and therefore the widths shown in Figure $9 b$ are only schematic. The blobs, although similar, do show a slight difference in $h_{m} F_{2}$, i.e., the two hard precipitation blobs $X_{1}$ and $X_{4}$ have $h_{m} F_{2}$ values about $20 \mathrm{~km}$ lower than for the soft precipitation blobs or the adjacent plasma. However, these differences in $h_{m} F_{2}$ are too small to be experimentally significant. A comparison of the relative blob densities in Figures $8 b$ and $9 b$ shows that the transport process has left the blobs relatively unchanged in going from $80^{\circ}$ to $70^{\circ}$ latitude. Notice, however, the blobs seen at Chatanika at $80^{\circ}$ magnetic latitude take about 30-50 min to reach $70^{\circ}$ and hence would be observed at a location to the west of Chatanika. The main conclusion to be drawn from the comparison of Figures $8 b$ and $9 b$ is that the source mechanism for blob formation cannot be determined after the blobs leave the source region.

\section{SUMMARY}

Our HLTD simulations of blob formation and decay lead us to conclude that in the auroral and polar ionosphere density irregularities on a spatial scale greater than $10 \mathrm{~km}$ should be the rule rather than the exception. A density irregularity (enhancement) is produced within a few minutes of a plasma flux tube entering a region of auroral ionization. This general time scale is consistent with the earlier work of Knudsen [1974]. Once formed, these blobs can persist for many hours, since their density decays at about the same rate as the adjacent lower density regions. Blobs are formed by any form of auroral precipitation and are removed only when a new ionization source produces densities an order of magnitude greater (i.e., sunlight).

The following quantitative conclusions were obtained from this study,

1. Observed precipitation energy fluxes can easily produce a factor of 2 increase in $N_{m} F_{2}$ in 10-15 min. This factor of 2 can be reduced by decreasing the energy flux or hardening the energy spectrum. In addition, if the plasma convection speed is increased or the arc length is reduced, the factor also decreases. Blobs of a few 10's of percent above background density can be easily expected from variations in all these parameters.

2. Hard $(\gtrsim 2 \mathrm{KeV})$ precipitation produces blobs whose ultimate $N_{m} F_{2}$ value depends on the density at the nominal "no precipitation" $h_{m} F_{2}$ altitude at the time when the plasma flux tube leaves the auroral region. After leaving the auroral production region, downward diffusion of plasma is the major low altitude maintenance mechanism in our model $(\sim 150 \mathrm{Km})$.

3. Soft $(\leqslant 200 \mathrm{eV})$ precipitation produces blobs whose $N_{m} F_{2}$ value depends on the $N_{m} F_{2}$ value in the source region if the precipitation-induced $h_{m} F_{2}$ altitude is above the nominal no precipitation $h_{m} F_{2}$ altitude. Downward diffusion is capable of transporting all the ionization produced at high altitudes down to the nominal $h_{m} F_{2}$ altitude in about 5-10 $\mathrm{min}$ after the flux tube leaves the precipitation region.

4. The blob $h_{m} F_{2}$ can only be used as an indicator of the hardness or softness of precipitation in the precipitation region. As soon as a blob is convected out of the source region, $h_{m} F_{2}$ rapidly (few minutes) adjusts to the value in the surrounding

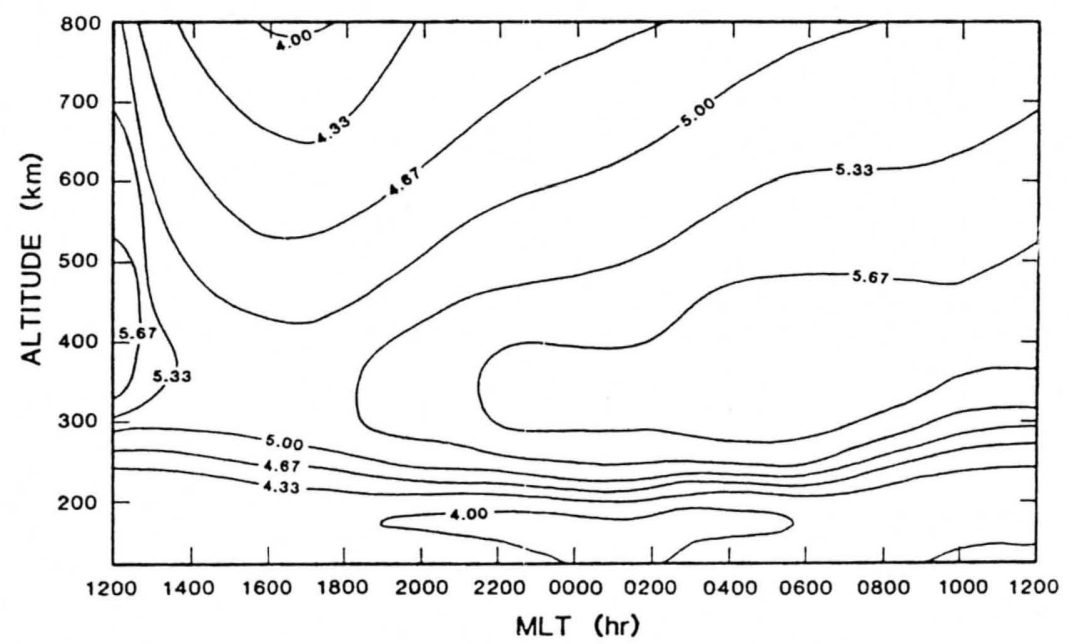

Fig. 9a. Contours of $\log _{10} N_{e}\left(\mathrm{~cm}^{-3}\right)$ as a function of altitude and MLT for the Chatanika geographic meridian but at a dipole latitude of $80^{\circ}$. This is the pattern that occurs in the absence of enhanced polar cap auroral precipitation. 


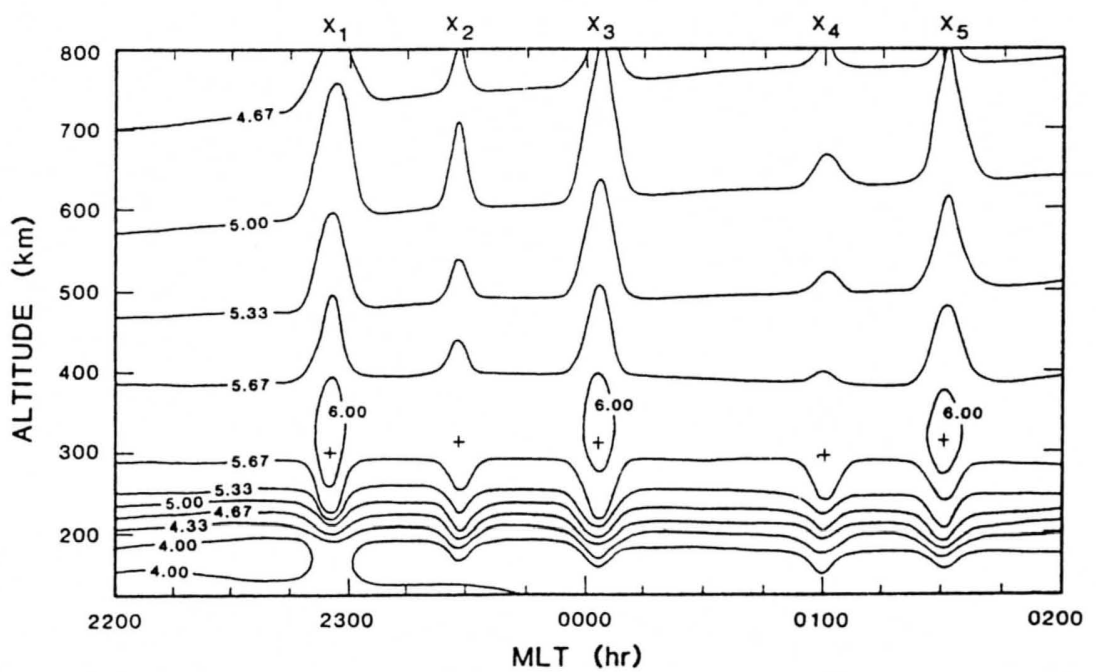

Fig. $9 b$. Contours of $\log _{10} N_{e}\left(\mathrm{~cm}^{-3}\right)$ as a function of altitude and MLT (between 2200-0200 hours) for the same location as in Figure $9 a$. For these calculations the enhanced polar cap auroral precipitation shown in Figure 7 has been included. The labels $X_{1}$ to $X_{5}$ identify five density enhancements. The crosses indicate the values of $h_{m} F_{2}$ in the density enhancements.

plasma. In section 3 of this study very low $h_{m} F_{2}$ values for nightside blobs were obtained due to the strong downward ExB induced drifts in our adopted convection model. If instead, strong neutral winds were present, the associated upward ion drifts would have produced high $h_{m} F_{2}$ values [Sojka and Schunk, 1985]. This change in $h_{m} F_{2}$ would have little effect on the overall blob characteristics.

5. The ratio of blob $N_{m} F_{2}$ to the adjacent nonblob $N_{m} F_{2}$ is maintained for hours or until the plasma flux tubes enter a new region of ionization, i.e., cross the sunlight terminator.

The model results also have a direct bearing on the interpretation of blob observations. In order to determine how an observed density irregularity was formed, it is necessary to know the past history of the plasma flux tube since blobs can survive for many hours in the night sector. A blob profile can only give information about the production precipitation spectrum when other evidence shows that the precipitation is occurring at the time the blob is observed. Hence, the conclusions of Vickrey et al. [1980] and Kelley et al. [1982] concerning the importance of soft precipitation for blob formation are difficult to prove. Indeed, our study suggests that any structured auroral form will give rise to blobs, the magnitude of which depends on the density enhancement at the $F$ peak for hard precipitation and on the density above the peak for soft precipitation.

From polar cap observations, Weber and Buchau [1981] concluded that the blob dimensions were related to the size and duration of auroral sun-aligned patches observed in the polar cap. Our study supports this conclusion and further indicates that the width of the patches transverse to their convection motion will vary drastically once convected away from the source, being narrow in throat regions and relatively wide in low convection polar cap regions (see section 3). More recent observations by Weber et al. [1984] show large scale blobs convecting antisunward in the polar cap with no local correlation to auroral precipitation. These observations as well as those of de la Beaujardiere [1985] demonstrate how plasma convection can readily transport blobs from their source. In both these cases the blob formation is associated with dayside solar EUV production, cleft production or some undefined enhanced plasma loss mechanism rather than the sun-aligned arcs used in section 3 .
A further problem with the interpretation of blob observations arises because of the role played by vertical transport which, through neutral wind and convection-induced ion drifts, can appreciably change the local plasma decay rate. Enhanced downward or upward induced ion drifts in local regions of high convection electric fields could be a major source of structuring for transpolar cap blobs. With this mechanism the blobs are formed in regions where no enhanced downward drifts occur and the lower density regions between blobs occur because of the electric field-induced lowering of the $F$ layer into a region of faster recombination rates. Since the blobs and the spaces between them are quite small, 10's of kilometer in the region of high electric fields, the identification of regions where locally strong ExB drifts exist is difficult. The spatial resolution of the incoherent scatter radars is on a comparable, if not poorer scale and hence makes this observation particularly insensitive. Kelley and Vickrey [1984] further point out that in regions of high electric fields the chemical $\mathrm{O}^{+}$loss rate increases, leading to a structuring in the $F$ region plasma.

Foster and Doupnik [1984] have inferred that significantly enhanced density blobs convect into the polar cap at the dayside throat region. These results would imply an ionization source for the blobs as opposed to large regions of downward drift leaving a smaller region of uneroded density (the blob). Indeed, the predominant orientation of the noon sector convection electric field causes both a poleward ExB drift and an upwardinduced plasma drift.

Based on the model results presented in this and our previous study [Sojka and Schunk, 1984], both blob formation mechanisms are feasible, and indeed, a combination of both may be at work. With improved auroral inputs and zonal electric field observations, our HLTD model would be better able to resolve the relative importance of plasma convection effects versus auroral production.

Acknowledgments. This research was supported by NASA grant NAGW-77 and NSF grant ATM-8417880 to Utah State University. The computer modeling effort was supported by the National Center for Atmospheric Research, which is sponsored by the National Science Foundation.

The Editor thanks J. F. Vickrey and another referee for their assistance in evaluating this paper. 


\section{REFERENCES}

Banks, P. M., C. R. Chappell, and A. F. Nagy, A new model for the interaction of auroral electrons with the atmosphere: Spectral degradation, backscatter, optical emission and ionization, J. Geophys. Res., 79, 1459-1470, 1974.

de la Beaujardiere, O., J. D. Craven, V. B. Wickwar, G. Candal, J. M. Holt, L. A. Frank, L. H. Brace, D. S. Evans, and J. D. Winningham, Universal time dependence of nighttime $F$ region densities at high latitudes, J. Geophys. Res., 90, 4319-4332, 1985.

Foster, J. C., and J. R. Burrows, Electron fluxes over the polar cap, 1, intense $\mathrm{KeV}$ fluxes during poststorm quieting, J. Geophys. Res., 81, 6016-6028, 1976.

Foster, J. C., and J. R. Doupnik, Plasma convection in the vicinity of the dayside cleft, J. Geophys. Res., 89, 9107-9113, 1984.

Heelis, R. A., W. B. Hanson, and J. L. Burch, Ion convection velocity reversals in the dayside cleft, J. Geophys. Res., 81, 3803-3809, 1976. Heelis, R. A., J. K. Lowell, and R. W. Spiro, A model of the highlatitude ionospheric convection pattern, J. Geophys. Res., 87, 63396345, 1982.

Kelly, J. D., and J. F. Vickrey, F-region ionospheric structure associated with antisunward flow near the dayside polar cusp, Geophys. Res. Lett., 11, 907-910, 1984.

Kelley, M. C., J. F. Vickrey, C. W. Carlson, and R. Torbert, On the origin and spatial extent of high-latitude $F$ region irregularities, $J$. Geophys. Res., 87, 4469-4475, 1982.

Knudsen, W. C., Magnetospheric convection and the high-latitude $F_{2}$ ionosphere, J. Geophys. Res., 79, 1046-1055, 1974.

Knudsen, W. C., P. M. Banks, J. D. Winningham, and D. M. Klumpar, Numerical model of the convecting $F_{2}$ ionosphere at high latitudes, $J$. Geophys. Res., 82, 4784-4792, 1977.

Meng, C.-I., Electron precipitation in the midday auroral oval, J. Geophys. Res., 86, 2149-2174, 1981.

Meng, C.-I., and S.-I. Akasofu, The relation between the polar cap auroral arc and the auroral oval arc, J. Geophys. Res., 81, 4004-4006, 1976.

Rees, M. H., Auroral ionization and excitation by incident energetic electrons, Planet. Space Sci., 11, 1209, 1963.

Rees, M. H., Auroral electrons, Space Sci., Rev., 10, 413, 1969.

Rees, M. H., and K. Maeda, Auroral electron spectra, J. Geophys. Res., 78, 8391, 1973.
Reiff, P. H., J. L. Burch, and R. A. Heelis, Dayside auroral arcs and convection, Geophys. Res. Lett., 5, 391-394, 1978.

Robinson, R. M., R. T. Tsunoda, J. F. Vickrey, and L. Guerin, Sources of $F$ region ionization enhancements in the nighttime auroral zones, Eos Trans. AGU, 66, 452, 1985.

Schunk, R. W., and W. J. Raitt, Atomic nitrogen and oxygen ions in the daytime high-latitude F region, J. Geophys. Res., 85, 1255-1272, 1980.

Snyder, A. L., and S.-I. Akasofu, Auroral oval photographs from the DMSP 8531 and 10533 Satellites, J. Geophys. Res., 81, 1799-1804, 1976.

Sojka, J. J., W. J. Raitt, and R. W. Schunk, A theoretical study of the high-latitude winter $F$ region at solar minimum for low magnetic activity, J. Geophys. Res., 86, 609-621, $1981 a$.

Sojka, J. J., W. J. Raitt, and R. W. Schunk, Plasma density features associated with strong convection in the winter high-latitude $F$ region, J. Geophys. Res., 86, 6908-6916, $1981 b$.

Sojka, J. J., and R. W. Schunk, A theoretical study of the high-latitude $F$ region's response to magnetospheric storm inputs, J. Geophys. Res., $88,2112-2122,1983$.

Sojka, J. J., and R. W. Schunk, Theoretical study of anomalously high $F$ region peak altitudes in the polar ionosphere, J. Geophys. Res., 90, 7525-7532, 1985.

Spiro, R. W., P. H. Reiff, and L. J. Maher, Precipitating electron energy flux and auroral zone conductances-An empirical model, J. Geophys. Res., 87, 8215-8227, 1982.

Vickrey, J. F., C. L. Rino, and T. A. Potemra, Chatanika/TRIAD observations of unstable ionization enhancements in the auroral $F$ region, Geophys. Res. Lett., 7, 789-792, 1980.

Weber, E. J., and J. Buchau, Polar cap F layer auroras, Geophys. Res. Lett., 8, 125-128, 1981.

Weber, E. J., J. Bachau, J. G. Moore, J. R. Sharber, R. C. Livingston, J. D. Winningham, and B. W. Reinisch, $F$ layer ionization patches in the polar cap, J. Geophys. Res., 89, 1683-1694, 1984.

R. W. Schunk and J. J. Sojka, Center for Atmospheric and Space Sciences, Utah State University, Logan, UT 84322-3400.

(Received May 3, 1985;

revised July 24, 1985;

accepted August 1, 1985.) 